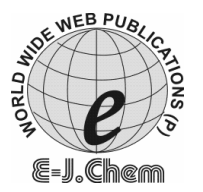

http://www.e-journals.net
CODEN ECJHAO

E-Journal of Chemistry

Vol. 3, No.1, pp 28-34, January 2006

\title{
Synthetic studies of Alkoxy isoindole-1,3-diones tetra- azabenzo[f]azulenes and their Antibacterial activity
}

\author{
M. AHMED, R. SHARMA, D. P. NAGDA, \\ V. K. SALVI and G. L. TALESARA* \\ Synthetic Organic Chemistry Research Laboratory \\ Department of Chemistry \\ M. L. Sukhadia University \\ Udaipur (Raj)-313 001 (India) \\ E-mail: gtalesara@yahoo.com,maqchem@yahoo.co.in
}

Received 2 October 2005; Accepted 23 January 2006

\begin{abstract}
Methylpyrazol-5-one 3 reacts with substituted benzaldehydes 4a-d in the presence of anhydrous sodium acetate to produce the corresponding 4arylidene-5-methyl-2,4-dihydro-pyrazol-3-ones 5a-d and the condensation of 5a-d with 2-bromoalkoxy-1H-isoindole-1, 3-(2H)-diones 2a-c furnished corresponding 2-[2-(4-arylidene-3-methyl-5-oxo-4,5-dihydro-pyrazol-1-yl)alkoxy]-isoindole-1,3diones 6a-l, which on cyclisation with o-phenylendiamine give titled compounds 7a-l. All the synthesized compounds have been characterized by elemental analysis and spectral data and screened for their antibacterial properties against various bacterial strains in order to obtain chemotherapeutic properties.
\end{abstract}

Key words: . Synthetic studies, antibacterial activity

\section{Introduction}

A great deal of synthesis work has been done in last few years on various compounds having a condensed diazepine nucleus, especially benzodiazepin have attracted the attention of several chemist and biologists because of their valuable therapeutic properties like tranquillizer $^{1-3}$, neuroleptic agents ${ }^{4}$, antineoplastic ${ }^{5,6}$, HIV-1 RT inhibitors ${ }^{7,8}$ antileukemic $^{9}$ and antifungal ${ }^{10}$ etc. It is interesting to note that pyrazoles are also reported as well known pharmacophores ${ }^{11-14}$. As a part of our studies aimed to synthesis of various heterocyclic imidoxy derivatives and in continuation to our work ${ }^{15-18}$ it seems interesting to integrate 
structural features from different biological active compounds in a single molecule. Consequently there have been many efforts for designing of novel, hybrid molecules possessing significant bioactivity. In the present work, we report the synthetic efforts to 29

G. L. TALESARA et al

synthesize alkoxy isoindole-1,3-dionestetra-azabenzo[f]azulenes containing benzodiazepine, pyrazole and imidoxy moieties.

\section{Experimental}

Melting points were determined in open capillary tubes and are uncorrected. The time required for completion of the reaction was monitored by TLC using Silica gel-G plates and spots were exposed in iodine chamber. IR spectra were recorded on a Perkin Elmer 1800 (FTIR) spectrometer ${ }^{1} \mathrm{H}$ NMR spectra $\left(\mathrm{CDCl}_{3}\right)$ were taken on a DRX-300 spectrometer (300 $\mathrm{MHz}$ ) using TMS as internal standard and chemical shifts are expressed in $\delta \mathrm{ppm}$. Mass spectra were taken on a jeol SX-102/DA-6000 spectrometer. 2-Hydroxy-1H-isoindole-1,3dione $^{20}$ 1, 2-Bromoalkoxy-1H-isoindole-1,3-(2H)-diones ${ }^{21}$ 2a-c and 3-methylpyrazol-5-one ${ }^{22}$ $\mathbf{3}$ were prepared by the reported methods.

Synthesis of 4-(4'-methoxybenzylidene)-5-methyl-2,4-dihydro-pyrazol-3-one 5a.

A mixture of 3-methylpyrazol-5-one 3 (0.98 g, 0.01 mole), anisaldehyde $\mathbf{4 a}(1.12 \mathrm{ml}, 0.01$ mole) and anhydrous sodium acetate $(0.82 \mathrm{~g}, 0.01 \mathrm{~mole})$ were suspended in acetic acid (30 $\mathrm{ml}$ ) and refluxed for 10 hours. The reaction mixture was filtered and the filtrate was poured on crushed ice. The solid obtained, was crystallised from ethanol. Compounds $\mathbf{5 b}$-d were also synthesized by the similar method using appropriate reactants with required change in reflux time.

5a: (c.f. Table 2); IR (KBr)(cm-1): $3435(\mathrm{~N}-\mathrm{H}), 3076(\mathrm{Ar}-\mathrm{H}), 2964\left(\mathrm{C}-\mathrm{H}, \mathrm{CH}_{3}\right), 1690(\mathrm{C}=\mathrm{O})$, $1602(\mathrm{C}=\mathrm{N}) ;{ }^{1} \mathbf{H}$ NMR $\left(\mathbf{C D C l}_{3}\right): 8.2(\mathrm{~s}, 1 \mathrm{H}, \mathrm{N}-\mathrm{H}), 7.4-7.0(\mathrm{~m}, 4 \mathrm{H}, \mathrm{Ar}-\mathrm{H}), 6.1(\mathrm{~s}, 1 \mathrm{H}, \mathrm{C}=\mathrm{CH})$, $3.7\left(\mathrm{~s}, 3 \mathrm{H}, \mathrm{OCH}_{3}\right), 1.7\left(\mathrm{~s}, 3 \mathrm{H}, \mathrm{CH}_{3}\right) . \mathbf{5 b} ; \mathbf{I R}(\mathbf{K B r})\left(\mathbf{c m}^{-1}\right): 3412(\mathrm{~N}-\mathrm{H}), 3080(\mathrm{Ar}-\mathrm{H}), 2952$ $\left(\mathrm{C}-\mathrm{H}, \mathrm{CH}_{3}\right), 1718(\mathrm{C}=\mathrm{O}), 1612(\mathrm{C}=\mathrm{N}), 728(\mathrm{Ar}-\mathrm{Cl}) ;{ }^{1} \mathbf{H} \mathbf{N M R}\left(\mathbf{C D C l}_{3}\right): 8.0(\mathrm{~s}, 1 \mathrm{H}, \mathrm{N}-\mathrm{H})$, 7.3-6.9 (m, 4H, Ar-H), $6.2(\mathrm{~s}, 1 \mathrm{H}, \mathrm{C}=\mathrm{CH}), 1.8\left(\mathrm{~s}, 3 \mathrm{H}, \mathrm{CH}_{3}\right) . \mathbf{5 c} ; \mathbf{I R}(\mathbf{K B r})\left(\mathbf{c m}^{-1}\right): 3396(\mathrm{~N}-$ $\mathrm{H}), 3062(\mathrm{Ar}-\mathrm{H}), 2946\left(\mathrm{C}-\mathrm{H}, \mathrm{CH}_{3}\right), 1705(\mathrm{C}=\mathrm{O}), 1612(\mathrm{C}=\mathrm{N}) ;{ }^{1} \mathbf{H} \mathbf{N M R}\left(\mathbf{C D C l}_{3}\right): 8.1$ (s, $1 \mathrm{H}, \mathrm{N}-\mathrm{H}), 7.4-7.1(\mathrm{~m}, 5 \mathrm{H}, \mathrm{Ar}-\mathrm{H}), 6.3(\mathrm{~s}, 1 \mathrm{H}, \mathrm{C}=\mathrm{CH}), 1.8\left(\mathrm{~s}, 3 \mathrm{H}, \mathrm{CH}_{3}\right) . \mathbf{5 d}$; IR $(\mathbf{K B r})\left(\mathbf{c m}^{-1}\right)$ : $3410(\mathrm{~N}-\mathrm{H}), 3070(\mathrm{Ar}-\mathrm{H}), 2950\left(\mathrm{C}-\mathrm{H}, \mathrm{CH}_{3}\right), 1710(\mathrm{C}=\mathrm{O}), 1626(\mathrm{C}=\mathrm{N}) ;{ }^{1} \mathbf{H} \mathbf{N M R}\left(\mathbf{C D C l}_{3}\right)$ : $8.1(\mathrm{~s}, 1 \mathrm{H}, \mathrm{N}-\mathrm{H}), 7.6-7.2(\mathrm{~m}, 4 \mathrm{H}, \mathrm{Ar}-\mathrm{H}), 6.2(\mathrm{~s}, 1 \mathrm{H}, \mathrm{C}=\mathrm{CH}), 2.0\left(\mathrm{~s}, 3 \mathrm{H}, \mathrm{CH}_{3}\right), 1.7(\mathrm{~s}, 3 \mathrm{H}$, pyrozole- $\left.\mathrm{CH}_{3}\right)$.

Synthesis of 2-[2-\{4-(4'-methoxybenzylidene)-3-methyl-5-oxo-4,5-dihydro-pyrazol1-yl\} ethoxy]-isoindol-1,3-dione 6a.

A mixture of compound 5a (2.16 g, 0.01 mole), with 2-bromoethoxy-1H-isoindole-1, 3(2H)-dione 2a (2.70 g, 0.01 mole) and pyridine $(2 \mathrm{ml})$ were refluxed in absolute alcohol (30 $\mathrm{ml})$ for $11 \mathrm{hrs}$. Excess of the solvent from the filtrate was removed under reduced pressure. On cooling, the solid separated was crystallized from ethanol. Compounds $6 \mathbf{b}-\mathbf{l}$ were also synthesized by the similar method using appropriate reactants and minor modification in reaction conditions.

6a: (c.f. Table 2); IR (KBr) (cm $\left.{ }^{-1}\right)$ : $3072(\mathrm{Ar}-\mathrm{H}), 2932\left(\mathrm{C}-\mathrm{H}, \mathrm{CH}_{3}\right), 2837\left(\mathrm{C}-\mathrm{H}, \mathrm{CH}_{2}\right), 1755$ $(\mathrm{C}=\mathrm{O}, \mathrm{CO}-\mathrm{N}-\mathrm{CO}), 1722(\mathrm{C}=\mathrm{O}$, pyrazole ring $), 1603(\mathrm{C}=\mathrm{N}), 1270(\mathrm{C}-\mathrm{N}) ;{ }^{1} \mathbf{H}$ NMR $\left(\mathbf{C D C l}_{3}\right)$ : 7.6-7.0 (m, 8H, Ar-H) 6.7, (s, $1 \mathrm{H}, \mathrm{C}=\mathrm{CH}), 3.6\left(\mathrm{~s}, 3 \mathrm{H}, \mathrm{OCH}_{3}\right), 3.4\left(\mathrm{t}, 2 \mathrm{H}, \mathrm{OCH}_{2} \mathrm{CH}_{2} \mathrm{~N}\right), 2.8$ (t, $\left.2 \mathrm{H}, \mathrm{OCH}_{2} \mathrm{CH}_{2} \mathrm{~N}\right), 1.9$ (s, 3H, $\left.\mathrm{CH}_{3}\right)$. 6c: (c.f. Table 2); $\mathbf{I R}(\mathbf{K B r})\left(\mathbf{c m}^{-1}\right): 3068(\mathrm{Ar}-\mathrm{H})$, $2946\left(\mathrm{C}-\mathrm{H}, \mathrm{CH}_{3}\right), 2848\left(\mathrm{C}-\mathrm{H}, \mathrm{CH}_{2}\right), 1776(\mathrm{CO}-\mathrm{N}-\mathrm{CO}), 1698(\mathrm{C}=\mathrm{O}$, pyrazole ring), 1620 $(\mathrm{C}=\mathrm{N}), 1280(\mathrm{C}-\mathrm{N}) ;{ }^{1} \mathbf{H}$ NMR $\left(\mathbf{C D C l}_{3}\right)$ : 7.7-7.2 (m, 9H, Ar-H), $6.8(\mathrm{~s}, 1 \mathrm{H}, \mathrm{C}=\mathrm{CH}), 3.4(\mathrm{t}$, 
$2 \mathrm{H}, \mathrm{OCH}_{2} \mathrm{CH}_{2} \mathrm{~N}$ ), $2.8\left(\mathrm{t}, 2 \mathrm{H}, \mathrm{OCH}_{2} \mathrm{CH}_{2} \mathrm{~N}\right), 1.9$ (s, 3H, $\left.\mathrm{CH}_{3}\right)$. 6f: (c.f. Table 2); IR (KBr) $\left(\mathbf{c m}^{-1}\right)$ : $3066(\mathrm{Ar}-\mathrm{H}), 2940\left(\mathrm{C}-\mathrm{H}, \mathrm{CH}_{3}\right), 2842\left(\mathrm{C}-\mathrm{H}, \mathrm{CH}_{2}\right), 1770(\mathrm{CO}-\mathrm{N}-\mathrm{CO}), 1705(\mathrm{C}=\mathrm{O}$, pyrazole ring), $1616(\mathrm{C}=\mathrm{N}), 1283(\mathrm{C}-\mathrm{N}), 746(\mathrm{Ar}-\mathrm{Cl}) ;{ }^{1} \mathbf{H}$ NMR $\left(\mathbf{C D C l}_{3}\right)$ : 7.5-7.1 (m, 8H,

Synthetic and their antibacterial activity studies

Ar-H), $6.6(\mathrm{~s}, 1 \mathrm{H}, \mathrm{C}=\mathrm{CH}), 3.5\left(\mathrm{t}, 2 \mathrm{H}, \mathrm{OCH}_{2} \mathrm{CH}_{2} \mathrm{CH}_{2} \mathrm{~N}\right), 2.8\left(\mathrm{t}, 2 \mathrm{H}, \mathrm{OCH}_{2} \mathrm{CH}_{2} \mathrm{CH}_{2} \mathrm{~N}\right), 2.5$ (quint, $\left.2 \mathrm{H}, \mathrm{OCH}_{2} \mathrm{CH}_{2} \mathrm{CH}_{2} \mathrm{~N}\right), 1.8\left(\mathrm{~s}, 3 \mathrm{H}, \mathrm{CH}_{3}\right)$. 6l: (c.f. Table 2); IR (KBr) $\left(\mathbf{c m}^{-1}\right)$ : 3076 (Ar-H), $2950\left(\mathrm{C}-\mathrm{H}, \mathrm{CH}_{3}\right), 2838\left(\mathrm{C}-\mathrm{H}, \mathrm{CH}_{2}\right), 1750(\mathrm{CO}-\mathrm{N}-\mathrm{CO}), 1700(\mathrm{C}=\mathrm{O}$ pyrazole ring), $1602(\mathrm{C}=\mathrm{N}), 1286(\mathrm{C}-\mathrm{N}) ;{ }^{1} \mathbf{H}$ NMR $\boldsymbol{\delta}\left(\mathbf{C D C l}_{3}\right): 7.3-6.8(\mathrm{~m}, 8 \mathrm{H}, \mathrm{Ar}-\mathrm{H}), 6.7(\mathrm{~s}, 1 \mathrm{H}, \mathrm{C}=\mathrm{CH}-$ $\mathrm{Ar}), 3.4\left(\mathrm{t}, 2 \mathrm{H}, \mathrm{OCH}_{2} \mathrm{CH}_{2} \mathrm{CH}_{2} \mathrm{CH}_{2} \mathrm{~N}\right), 2.8\left(\mathrm{t}, 2 \mathrm{H}, \mathrm{OCH}_{2} \mathrm{CH}_{2} \mathrm{CH}_{2} \mathrm{CH}_{2} \mathrm{~N}\right), 2.2\left(\mathrm{~s}, 3 \mathrm{H}, \mathrm{CH}_{3}\right), 1.8$ (s, $3 \mathrm{H}$, Pyrazole $\mathrm{CH}_{3}$ ).

Synthesis of 2-[2-\{10-(4'-methoxyphenyl)-1-methyl-10,10a-dihydro-9H-2,3,4,9tetra-azabenzo [f] azulen-3-yl\}ethoxy]-isoindol-1,3-dione 7a.

Compound 6a (4.05 g, 0.01 mole) was dissolved in $80 \mathrm{ml}$ hot xylene in round bottomed flask and o-phenylenediamine $(1.08 \mathrm{~g}, 0.01 \mathrm{~mole})$ was also dissolved in $20 \mathrm{ml}$ boiling xylene. Now the boiling solution of o-phenylenediamine was added in small installments to the solution of 6a and refluxed for 8-10 h. During the reaction water formed was separated by Dean-stark apparatus. Excess of solvent was then removed under reduced pressure. The residue was titurated with light petroleum ether and benzene (9:1) to give final product. It was filtered and recrystallized from light peterolium benzene. Compounds $\mathbf{7 b}$-l were also synthesized by the similar method using appropriate reactants.

7a: (c.f. Table 2); IR (KBr) (cm $\left.{ }^{-1}\right)$ : $3432(\mathrm{~N}-\mathrm{H}), 3060(\mathrm{Ar}-\mathrm{H}), 2928\left(\mathrm{C}-\mathrm{H}, \mathrm{CH}_{3}\right), 2836(\mathrm{C}-\mathrm{H}$, $\left.\mathrm{CH}_{2}\right), 1750(\mathrm{CO}-\mathrm{N}-\mathrm{CO}), 1606(\mathrm{C}=\mathrm{N}), 1267(\mathrm{C}-\mathrm{N}) ;{ }^{1} \mathbf{H} \mathbf{~ N M R}\left(\mathbf{C D C l}_{3}\right)$ : 7.8-7.6 (m, 12H, Ar$\mathrm{H}), 4.8(\mathrm{~s}, 1 \mathrm{H}, \mathrm{NH}), 3.9\left(\mathrm{~s}, 3 \mathrm{H}, \mathrm{OCH}_{3}\right), 3.2\left(\mathrm{t}, 2 \mathrm{H}, \mathrm{OCH}_{2} \mathrm{CH}_{2} \mathrm{~N}\right), 2.7\left(\mathrm{t}, 2 \mathrm{H}, \mathrm{OCH}_{2} \mathrm{CH}_{2} \mathrm{~N}\right)$, $1.9\left(\mathrm{~s}, 3 \mathrm{H}, \mathrm{CH}_{3}\right) ; \mathrm{m} / \mathrm{z}: 493$ [M] $^{+}, 386,204,190,162,146,132,107,104,76.7 \mathrm{c}:$ (c.f. Table 2); IR (KBr) (cm $\left.{ }^{-1}\right)$ : $3410(\mathrm{~N}-\mathrm{H}), 3070(\mathrm{Ar}-\mathrm{H}), 2940\left(\mathrm{C}-\mathrm{H}, \mathrm{CH}_{3}\right), 2842\left(\mathrm{C}-\mathrm{H}, \mathrm{CH}_{2}\right), 1760$ $(\mathrm{CO}-\mathrm{N}-\mathrm{CO}), 1620(\mathrm{C}=\mathrm{N}), 1281(\mathrm{C}-\mathrm{N}) ;{ }^{1} \mathbf{H}$ NMR $\left(\mathbf{C D C l}_{3}\right)$ : 7.8-7.6 (m, 13H, Ar-H), $4.8(\mathrm{~s}$, $1 \mathrm{H}, \mathrm{NH}), 3.3\left(\mathrm{t}, 2 \mathrm{H}, \mathrm{OCH}_{2} \mathrm{CH}_{2} \mathrm{~N}\right), 2.8\left(\mathrm{t}, 2 \mathrm{H}, \mathrm{OCH}_{2} \mathrm{CH}_{2} \mathrm{~N}\right), 2.0\left(\mathrm{~s}, 3 \mathrm{H}, \mathrm{CH}_{3}\right) ; \mathrm{m} / \mathrm{z}: 463[\mathrm{M}]$ +., 387, 190, 162, 146, 132, 104, 76. 7f: (c.f. Table II); IR (KBr) $\left(\mathbf{c m}^{-1}\right): 3420(\mathrm{~N}-\mathrm{H}), 3068$ (Ar-H), $2932\left(\mathrm{C}-\mathrm{H}, \mathrm{CH}_{3}\right), 2830\left(\mathrm{C}-\mathrm{H}, \mathrm{CH}_{2}\right), 1742(\mathrm{CO}-\mathrm{N}-\mathrm{CO}), 1618(\mathrm{C}=\mathrm{N}), 1274(\mathrm{C}-\mathrm{N})$, $750(\mathrm{Ar}-\mathrm{Cl}) ;{ }^{1} \mathbf{H}$ NMR $\left(\mathbf{C D C l}_{3}\right)$ : 7.8-7.6 (m, 12H, Ar-H), $4.9(\mathrm{~s}, 1 \mathrm{H}, \mathrm{NH}), 3.3(\mathrm{t}, 2 \mathrm{H}$, $\mathrm{OCH}_{2} \mathrm{CH}_{2} \mathrm{CH}_{2} \mathrm{~N}$ ), 2.9 (t, $2 \mathrm{H}, \mathrm{OCH}_{2} \mathrm{CH}_{2} \mathrm{CH}_{2} \mathrm{~N}$ ), 2.6 (quint., $2 \mathrm{H}, \mathrm{OCH}_{2} \mathrm{CH}_{2} \mathrm{CH}_{2} \mathrm{~N}$ ), 2.1 (s, 3H, $\left.\mathrm{CH}_{3}\right) ; \mathrm{m} / \mathrm{z}: 513\left[\mathrm{M}^{+}+2\right]^{+}, 511[\mathrm{M}]^{+}, 204,190,162,146,132,104,76.7 \mathrm{l}$ : (c.f. Table 2); IR $(\mathrm{KBr})\left(\mathbf{c m}^{-1}\right): 3422(\mathrm{~N}-\mathrm{H}), 3062(\mathrm{Ar}-\mathrm{H}), 2942\left(\mathrm{C}-\mathrm{H}, \mathrm{CH}_{3}\right), 2848\left(\mathrm{C}-\mathrm{H}, \mathrm{CH}_{2}\right), 1756(\mathrm{CO}-\mathrm{N}-$ $\mathrm{CO}), 1610(\mathrm{C}=\mathrm{N}), 1283(\mathrm{C}-\mathrm{N}) ;{ }^{1} \mathrm{H}$ NMR $\left(\mathrm{CDCl}_{3}\right)$ : 7.7-7.6 (m, 12H, $\left.\mathrm{Ar}-\mathrm{H}\right), 4.7(\mathrm{~s}, 1 \mathrm{H}, \mathrm{NH})$, $3.7(\mathrm{~d}, 1 \mathrm{H}, \mathrm{CH}-\mathrm{Ar}), \quad 3.5(\mathrm{~d}, 1 \mathrm{H}, \mathrm{CH}), 3.2\left(\mathrm{t}, 2 \mathrm{H}, \quad \mathrm{OCH}_{2} \mathrm{CH}_{2} \mathrm{CH}_{2} \mathrm{CH}_{2} \mathrm{~N}\right), 2.8(\mathrm{t}, 2 \mathrm{H}$, $\mathrm{OCH}_{2} \mathrm{CH}_{2} \mathrm{CH}_{2} \mathrm{CH}_{2} \mathrm{~N}$ ), 2.3 (s, 3H, $\mathrm{CH}_{3}$ ), 1.8 (s, 3H, Pyrazole $\mathrm{CH}_{3}$ ); m/z: $505[\mathrm{M}]^{+}, 218,162$, $146,132,104,76$.

\section{Results and Discussion}

4-Arylidene-5-methyl-2,4-dihydro-pyrazol-3-ones 5a-d were synthesized by the reaction of 3-methylpyrazol-5-one 3 with substituted benzaldehydes 4 a-d in the presence of anhydrous sodium acetate and the formation of $\mathbf{5 a}$ was characterized by ${ }^{1} \mathrm{H}$ NMR signal at $\delta 6.1 \mathrm{ppm}$ for $\mathrm{C}=\mathrm{CH}-\mathrm{Ar}$. Compounds 5a-d were further condensed with 2-bromoalkoxy-1H-isoindole1,3-(2H)-dione 2a-c in the presence of pyridine to give 2-[2-(4-arylidene-3-methyl-5-oxo4,5-dihydro-pyrazol-1-yl)alkoxy]-isoindole-1,3-diones 6a-l. Absence of free stretching IR vibration band for $\mathrm{NH}$ group at $3435 \mathrm{~cm}^{-1}$ and ${ }^{1} \mathrm{H}$ NMR signal for the proton of $\mathrm{NH}$ group at $\delta 8.2 \mathrm{ppm}$, which was present in its precursor 5a-d confirms the formation of $\mathbf{6 a}-\mathbf{l}$. 
Compounds 6a-l were further cyclized with o-phenylenediamine in dry xylene to give final product 7a-l. Structural assignments of compounds $7 \mathbf{a}-\mathbf{l}$ are based on IR, ${ }^{1} \mathrm{H}$ NMR, Mass spectral data and elemental analysis.<smiles>O=C1c2ccccc2C(=O)N1O</smiles>

1
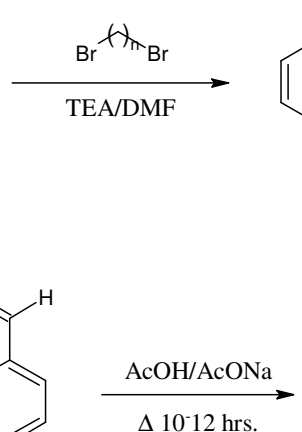<smiles>CC1=NNC(=O)C1</smiles><smiles>[R]c1ccc(C=O)cc1</smiles>

4a-d<smiles></smiles>

2a-c<smiles>Cc1nn(ON2C(=O)c3ccccc3C2=O)c2c1C(c1ccc(P)cc1)=Nc1ccccc1N2</smiles><smiles>Nc1ccccc1N</smiles><smiles>[R5]c1ccc(/C=C2/C(=O)NN=C2C)cc1</smiles>

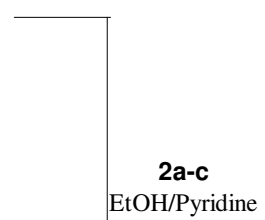

$\Delta 8^{-13} \mathrm{hrs}$.<smiles>[R]c1ccc(/C=C2/C(=O)N([Al]ON3C(=O)c4ccccc4C3=O)N=C2C)cc1</smiles>

Where $\mathrm{n}=2,3,4 ; \mathrm{R}=\mathrm{OCH}_{3}, \mathrm{Cl}, \mathrm{H}, \mathrm{CH}_{3}$

$\begin{array}{llllll}\text { a } & \mathrm{R}=\mathrm{OCH}_{3}, & \mathrm{n}=2 & \mathrm{~g} & \mathrm{R}=\mathrm{H}, & \mathrm{n}=3 \\ \text { b } & \mathrm{R}=\mathrm{Cl}, & \mathrm{n}=2 & \mathrm{~h} & \mathrm{R}=\mathrm{CH}_{3}, & \mathrm{n}=3 \\ \text { c } & \mathrm{R}=\mathrm{H}, & \mathrm{n}=2 & \mathrm{i} & \mathrm{R}=\mathrm{OCH}_{3}, & \mathrm{n}=4 \\ \text { d } & \mathrm{R}=\mathrm{CH}_{3}, & \mathrm{n}=2 & \text { j } & \mathrm{R}=\mathrm{Cl}, & \mathrm{n}=4 \\ \text { e } & \mathrm{R}=\mathrm{OCH}_{3}, & \mathrm{n}=3 & \mathrm{k} & \mathrm{R}=\mathrm{H}, & \mathrm{n}=4 \\ \text { f } & \mathrm{R}=\mathrm{Cl}, & \mathrm{n}=3 & \mathrm{l} & \mathrm{R}=\mathrm{CH}_{3}, & \mathrm{n}=4\end{array}$

\section{Antibacterial Screening}

In the present investigation all the synthesized compounds were screened at $100 \mu \mathrm{g} / \mathrm{ml}$ against various bacterial strains viz. Escherichia coli, Proteus mirabilis, Klebsiella pneumonia, Pseudomonas aureginosa, Salmonella typhi and Staphylococcus aureus for their antibacterial activity using amicacin as an standard drug by cup and well method developed 
by Collee, Fraser, Marmion and Simmons ${ }^{19}$. The screening results are summarized in Table 1 indicating that all the synthesized compounds have been manifested moderate to fairly good growth inhibition. Especially, compounds $7 \mathrm{~b}, 7 \mathrm{e}, 7 \mathrm{~g}, 7 \mathrm{i}, 7 \mathrm{j}$ and $7 \mathrm{k}$ have shown strong antibacterial activity as compared to standard.

Synthetic and their antibacterial activity studies

Table1. Antimicrobial activity of synthesized compounds 7a-1 Zone of inhibition (mm)/(activity index)

\begin{tabular}{|c|c|c|c|c|c|c|}
\hline \multirow[t]{2}{*}{ Compd. } & \multicolumn{6}{|c|}{ Antibacterial Activity $(100 \mu \mathrm{g} / \mathrm{ml})$} \\
\hline & $\begin{array}{c}\text { E. } \\
\text { coli }\end{array}$ & $\begin{array}{c}P . \\
\text { mirabilis }\end{array}$ & $\begin{array}{c}K . \\
\text { pneumonia }\end{array}$ & $\begin{array}{c}P . \\
\text { aureginosa }\end{array}$ & $\begin{array}{c}S . \\
\text { typhi }\end{array}$ & S. aureus \\
\hline $7 a$ & $17 / 1.06$ & $10 / 0.62$ & $11 / 0.84$ & $11 / 0.91$ & $10 / 0.50$ & $12 / 0.80$ \\
\hline $7 b$ & $13 / 0.81$ & $17 / 1.06$ & $15 / 1.15$ & $09 / 0.75$ & $16 / 0.80$ & $14 / 0.90$ \\
\hline $7 \mathrm{c}$ & $07 / 0.43$ & $09 / 0.56$ & $10 / 0.76$ & $07 / 0.58$ & $13 / 0.65$ & $05 / 0.33$ \\
\hline $7 d$ & $12 / 0.75$ & $07 / 0.43$ & 08/0.61 & $11 / 0.91$ & $09 / 0.45$ & $11 / 0.73$ \\
\hline $7 e$ & $15 / 0.93$ & $13 / 0.81$ & $11 / 0.84$ & $10 / 0.83$ & $18 / 0.90$ & $16 / 1.06$ \\
\hline $7 f$ & $09 / 0.56$ & $11 / 0.68$ & $10 / 0.76$ & $07 / 0.58$ & $13 / 0.65$ & $12 / 0.80$ \\
\hline $7 \mathrm{~g}$ & $07 / 0.43$ & $12 / 0.75$ & $07 / 0.53$ & $10 / 0.83$ & $09 / 0.45$ & $08 / 0.53$ \\
\hline $7 \mathrm{~h}$ & $13 / 0.81$ & $09 / 0.56$ & $09 / 0.69$ & 08/0.66 & $11 / 0.55$ & $06 / 0.40$ \\
\hline $7 \mathrm{i}$ & $14 / 0.87$ & $13 / 0.81$ & $11 / 0.84$ & $13 / 1.08$ & $09 / 0.45$ & $11 / 0.73$ \\
\hline $7 \mathrm{j}$ & $12 / 0.75$ & $08 / 0.50$ & $14 / 1.07$ & $09 / 0.75$ & $17 / 0.85$ & $13 / 0.86$ \\
\hline $7 \mathrm{k}$ & $06 / 0.37$ & $05 / 0.31$ & $11 / 0.84$ & $06 / 0.50$ & $08 / 0.40$ & $14 / 0.90$ \\
\hline 71 & $10 / 0.62$ & $12 / 0.75$ & $07 / 0.53$ & $09 / 0.75$ & $12 / 0.60$ & $09 / 0.60$ \\
\hline Stand. & 16 & 16 & 13 & 12 & 20 & 15 \\
\hline
\end{tabular}

(Activity index) = Inhibition zone of the sample/Inhibition zone of the standard.

Standard used: Amicacin

Table-2. Physical and analytical data of compounds 5a-d, 6a-1 and 7a-1.

\begin{tabular}{ccccccc}
\hline Compd. & $\begin{array}{c}\mathrm{m} . \mathrm{p} \\
\end{array}$ & Yield & Molecular & \multicolumn{3}{c}{ \% Found/Calcd } \\
\cline { 5 - 8 } & $(\%)$ & formula & $\mathrm{C}$ & $\mathrm{C}$ & $\mathrm{H}$ & $\mathrm{N}$ \\
\hline $5 \mathrm{a}$ & 168 & 62 & $\mathrm{C}_{12} \mathrm{H}_{12} \mathrm{~N}_{2} \mathrm{O}_{2}$ & $66.58 / 66.66$ & $5.49 / 5.55$ & $12.91 / 12.96$ \\
$5 \mathrm{~b}$ & 156 & 60 & $\mathrm{C}_{11} \mathrm{H}_{9} \mathrm{~N}_{2} \mathrm{OCl}$ & $59.74 / 59.86$ & $4.04 / 4.08$ & $12.72 / 12.69$ \\
$5 \mathrm{c}$ & 172 & 71 & $\mathrm{C}_{11} \mathrm{H}_{10} \mathrm{~N}_{2} \mathrm{O}$ & $70.92 / 70.96$ & $5.31 / 5.37$ & $15.01 / 15.05$ \\
$5 \mathrm{~d}$ & 166 & 66 & $\mathrm{C}_{12} \mathrm{H}_{12} \mathrm{~N}_{2} \mathrm{O}$ & $71.60 / 72.00$ & $5.70 / 6.00$ & $13.96 / 14.00$ \\
$6 \mathrm{a}$ & 130 & 60 & $\mathrm{C}_{22} \mathrm{H}_{19} \mathrm{~N}_{3} \mathrm{O}_{5}$ & $65.16 / 65.18$ & $4.52 / 4.69$ & $9.89 / 10.37$ \\
$6 \mathrm{~b}$ & 141 & 64 & $\mathrm{C}_{21} \mathrm{H}_{16} \mathrm{~N}_{3} \mathrm{O}_{4} \mathrm{Cl}$ & $61.48 / 61.53$ & $3.87 / 3.90$ & $10.19 / 10.26$ \\
$6 \mathrm{c}$ & 170 & 66 & $\mathrm{C}_{21} \mathrm{H}_{17} \mathrm{~N}_{3} \mathrm{O}_{4}$ & $67.12 / 67.20$ & $4.39 / 4.53$ & $11.09 / 11.20$ \\
$6 \mathrm{~d}$ & 120 & 60 & $\mathrm{C}_{22} \mathrm{H}_{19} \mathrm{~N}_{3} \mathrm{O}_{4}$ & $68.59 / 67.86$ & $4.81 / 4.88$ & $10.72 / 10.79$ \\
$6 \mathrm{e}$ & 146 & 69 & $\mathrm{C}_{23} \mathrm{H}_{21} \mathrm{~N}_{3} \mathrm{O}_{5}$ & $65.72 / 65.87$ & $5.00 / 5.01$ & $9.86 / 10.02$ \\
$6 \mathrm{f}$ & 159 & 64 & $\mathrm{C}_{22} \mathrm{H}_{18} \mathrm{~N}_{3} \mathrm{O}_{4} \mathrm{Cl}$ & $62.29 / 62.33$ & $4.14 / 4.25$ & $9.72 / 9.91$ \\
$6 \mathrm{~g}$ & 176 & 59 & $\mathrm{C}_{22} \mathrm{H}_{19} \mathrm{~N}_{3} \mathrm{O}_{4}$ & $67.75 / 67.86$ & $4.83 / 4.88$ & $10.54 / 10.79$ \\
$6 \mathrm{~h}$ & 144 & 61 & $\mathrm{C}_{23} \mathrm{H}_{21} \mathrm{~N}_{3} \mathrm{O}_{4}$ & $68.40 / 68.48$ & $5.11 / 5.21$ & $10.03 / 10.42$ \\
$6 \mathrm{i}$ & 153 & 61 & $\mathrm{C}_{24} \mathrm{H}_{23} \mathrm{~N}_{3} \mathrm{O}_{5}$ & $66.47 / 66.51$ & $5.29 / 5.31$ & $9.54 / 9.69$ \\
$6 \mathrm{j}$ & 182 & 68 & $\mathrm{C}_{23} \mathrm{H}_{20} \mathrm{~N}_{3} \mathrm{O}_{4} \mathrm{Cl}$ & $63.00 / 63.08$ & $4.34 / 4.57$ & $9.56 / 9.60$ \\
$6 \mathrm{k}$ & 186 & 65 & $\mathrm{C}_{23} \mathrm{H}_{21} \mathrm{~N}_{3} \mathrm{O}_{4}$ & $68.42 / 68.48$ & $5.19 / 5.21$ & $10.37 / 10.42$ \\
$6 \mathrm{l}$ & 194 & 56 & $\mathrm{C}_{24} \mathrm{H}_{23} \mathrm{~N}_{3} \mathrm{O}_{4}$ & $69.02 / 69.06$ & $5.31 / 5.51$ & $10.00 / 10.07$ \\
$7 \mathrm{a}$ & 268 & 58 & $\mathrm{C}_{28} \mathrm{H}_{23} \mathrm{~N}_{5} \mathrm{O}_{4}$ & $68.01 / 68.15$ & $4.59 / 4.66$ & $14.01 / 14.19$ \\
$7 \mathrm{~b}$ & 290 & 60 & $\mathrm{C}_{27} \mathrm{H}_{20} \mathrm{~N}_{5} \mathrm{O}_{3} \mathrm{Cl}$ & $64.95 / 65.12$ & $3.91 / 4.02$ & $13.92 / 14.07$ \\
$7 \mathrm{c}$ & 246 & 51 & $\mathrm{C}_{27} \mathrm{H}_{21} \mathrm{~N}_{5} \mathrm{O}_{3}$ & $69.82 / 69.97$ & $4.48 / 4.53$ & $15.02 / 15.11$ \\
$7 \mathrm{~d}$ & 305 & 52 & $\mathrm{C}_{28} \mathrm{H}_{23} \mathrm{~N}_{5} \mathrm{O}_{3}$ & $70.40 / 70.44$ & $4.75 / 4.82$ & $14.61 / 14.67$
\end{tabular}




$\begin{array}{rcccccc}7 \mathrm{e} & 273 & 61 & \mathrm{C}_{29} \mathrm{H}_{25} \mathrm{~N}_{5} \mathrm{O}_{4} & 68.55 / 68.63 & 4.87 / 4.93 & 13.71 / 13.80 \\ 7 \mathrm{f} & 287 & 56 & \mathrm{C}_{28} \mathrm{H}_{22} \mathrm{~N}_{5} \mathrm{O}_{3} \mathrm{Cl} & 65.61 / 65.68 & 4.23 / 4.30 & 13.61 / 13.68 \\ 7 \mathrm{~g} & 278 & 45 & \mathrm{C}_{28} \mathrm{H}_{23} \mathrm{~N}_{5} \mathrm{O}_{3} & 70.38 / 70.44 & 4.75 / 4.82 & 14.59 / 14.67 \\ 33 & \text { G. L. TALESARACLI }\end{array}$

\begin{tabular}{ccccccc}
\hline $7 \mathrm{~h}$ & 270 & 52 & $\mathrm{C}_{29} \mathrm{H}_{25} \mathrm{~N}_{5} \mathrm{O}_{3}$ & $70.79 / 70.87$ & $5.00 / 5.09$ & $14.21 / 14.25$ \\
$7 \mathrm{i}$ & 284 & 53 & $\mathrm{C}_{30} \mathrm{H}_{27} \mathrm{~N}_{5} \mathrm{O}_{3}$ & $69.03 / 69.09$ & $5.10 / 5.18$ & $13.37 / 13.43$ \\
$7 \mathrm{j}$ & 254 & 59 & $\mathrm{C}_{29} \mathrm{H}_{24} \mathrm{~N}_{5} \mathrm{O}_{3} \mathrm{Cl}$ & $66.16 / 66.22$ & $4.50 / 4.56$ & $13.23 / 13.32$ \\
$7 \mathrm{k}$ & 292 & 52 & $\mathrm{C}_{29} \mathrm{H}_{25} \mathrm{~N}_{5} \mathrm{O}_{3}$ & $70.81 / 70.87$ & $5.03 / 5.09$ & $14.19 / 14.25$ \\
71 & 315 & 58 & $\mathrm{C}_{30} \mathrm{H}_{29} \mathrm{~N}_{5} \mathrm{O}_{3}$ & $71.19 / 71.28$ & $5.29 / 5.34$ & $13.79 / 13.86$ \\
\hline
\end{tabular}

\section{Conclusion}

In the present paper we have succeeded to synthesize alkoxy isoindole-1,3-diones tetraazabenzo[f]azulenes in good yields which have been tested for antibacterial properties. Although compounds show moderate to good activity but some of the synthesized compounds have shown significant antibacterial activity which reveals the chemotherapeutic values of the synthetic compounds.

\section{Acknowledgement}

The author is thankful to Head, Department of Chemistry, M. L. Sukhadia University for providing the necessary laboratory facilities, Head, Microbial Laboratory, R. N. T. Medical College, Udaipur for providing facility for antibacterial evaluation and Head, RSIC, CDRI, Lucknow for spectral measurements and elemental analysis. One of the authors (DPN) is thankful to CSIR for providing necessary financial assistance.

\section{References}

1. Redenko G M , Voronina T A, Shatrova N G and Chepelev V M, Bull. Eksp. Bio. Med., 1982, 94(8), 54.

2. Fitzgerald J E, Delalgleria F A and Mc Guive E J, Fundam. Appl. Toxicol., 1984, 4, 178 .

3. Vida A, Medicinal Chemistry Part III, in M.W. Wolf \& A. Burger (Ed.), Jhon Wiley and Sons, New York 1981, pp.787.

4. Ellefson C R, Woo C M , Miller A and Kehr J R, J. Med. Chem., 1978, 21, 952.

5. Vora M M, Diss. Abstr. Int. (Sci.), 1979, 39, 3849.

6. Levin J L, Di Joseph J F, Killar L M, Sung A, Walter T, Sharr M A, Roth C E, Skotnicki J S and Albright J P, Bioorg. Med. Chem. Lett., 1998, 8 (19), 2972.

7. Cywin C L, Klunder J M and Huttox S E, J. Med. Chem., 1998, 41 (16), 2972.

8. Bellarosa D, Antohelli G and Declereq E, Antiviral Res., 1996, 30, 109.

9. Dal Piaz V, Ciciani G and Giovannoni M P, Farmaco., 1991, 46 ,435.

10. Majchrzak M, Mikolajewska H, Krakowiak K, Sikarska R and Kotelko B, Acta. Pol. Pharma, 1981,38, 545.

11. Coppe F T and Fawzi M M, J. Heterocycl. Chem., 1977, 34, 1693.

12. Steinmetz A and Tietze L F, Ger Offen. DE 19, 627, 002 (CL. CO7D231/22) 8 Jan. 1998, Appl. 19, 627, 002, 5 Jul 1996, 14pp, Chem. Abstr., 1998, 128, 114946e.

13. Suzuki Y, Takemura Y Y, Iwamoto K, Higashino T and Miyashito A, Chem. Pharm. Bull., 1996, 49, 199.

14. Bhagwat S S, Lee C, Cowart M D, Mckie J and Grillot A L, PCT Int. Appl. WO 9846, 605 (CL. CO7D471/04) 22 Oct. 1998, US Appl. 818, 216, 16 Apr. 1997 172pp; Chem. Abstr., 1996, 129, 316240b. 
15. Bhawani Singh, Lalit Kumar Baregama, Neha Dixit, Maqbool Ahmed and Talesara G L, Ind. J. Chem, 2005, 44(B).

Synthetic and their antibacterial activity studies

16. Bhawani Singh, Lalit Kumar Baregama, Ranjana Sharma, Maqbool Ahmed and Talesara G L, J. Ind. Chem. Soc., 2005, 82, 1.

17. Baregama L K, Ahmed M, Dak G, Sharma K and Talesara G L, Ind. J. Pharma., 2004, 36(5), 315.

18. Bhawani Singh, Deepika Mehta, Lalit K. Baregama and Talesara G L., Ind. J. Chem., 2004, 43B, 1306.

19. Collee G J, Fraser G A, Marmion P B and Simmons A, Practical Medical Microbiology, $14^{\text {th }}$ Ed. Vol. 11, Churchill Livingstone, Edinburg, 1996, 163.

20. Orndroff W.R and Pratt D S, Am. Chem. J., 1917, 47, 89.

21. Bauer L. \& Suresh K.S., J. Org. Chem., 28, 1963, 1604.

22. Vogel A.I., "A text book of Practical Organic Chemistry" Orient Longman (ELBS ed), 1989 pp. 807. 


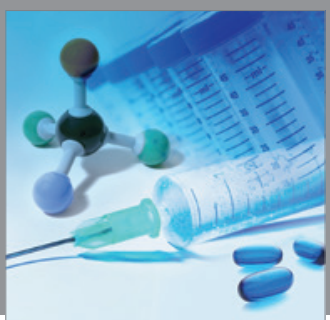

International Journal of

Medicinal Chemistry

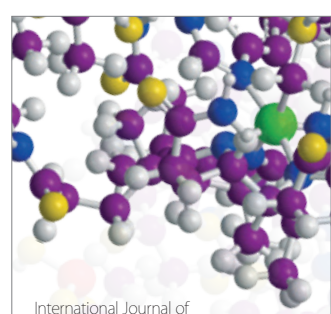

Carbohydrate Chemistry

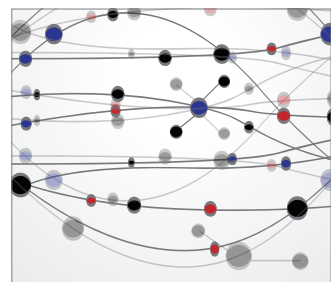

The Scientific World Journal
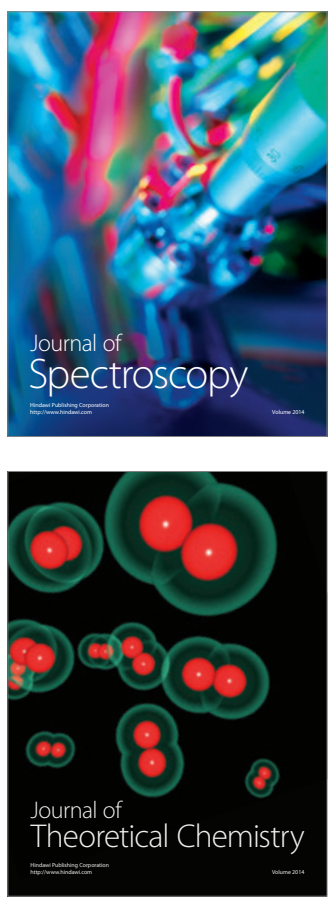
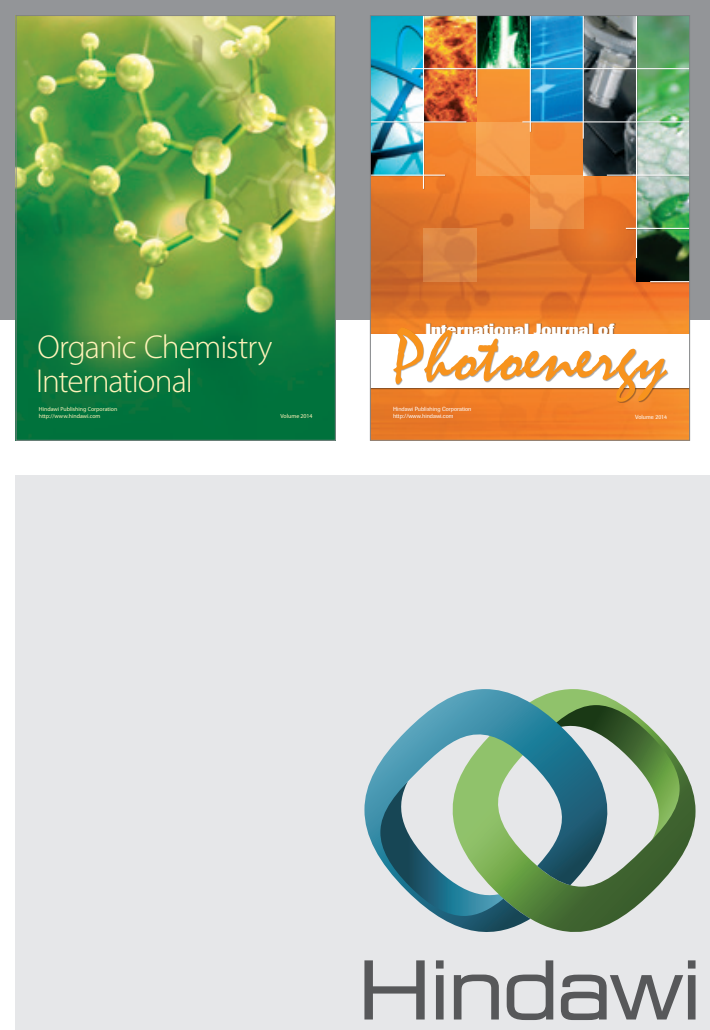

Submit your manuscripts at

http://www.hindawi.com
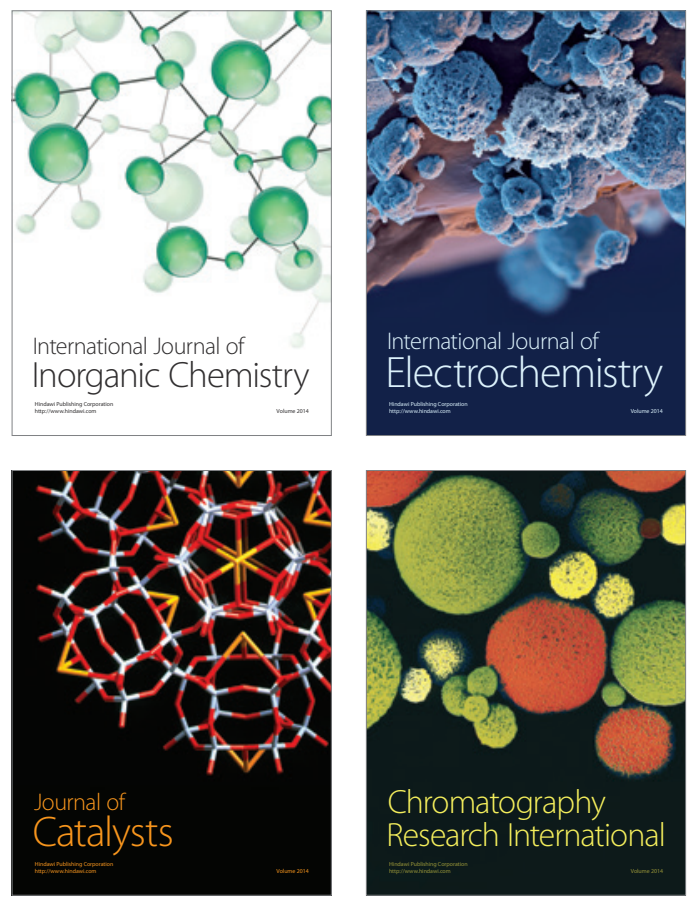
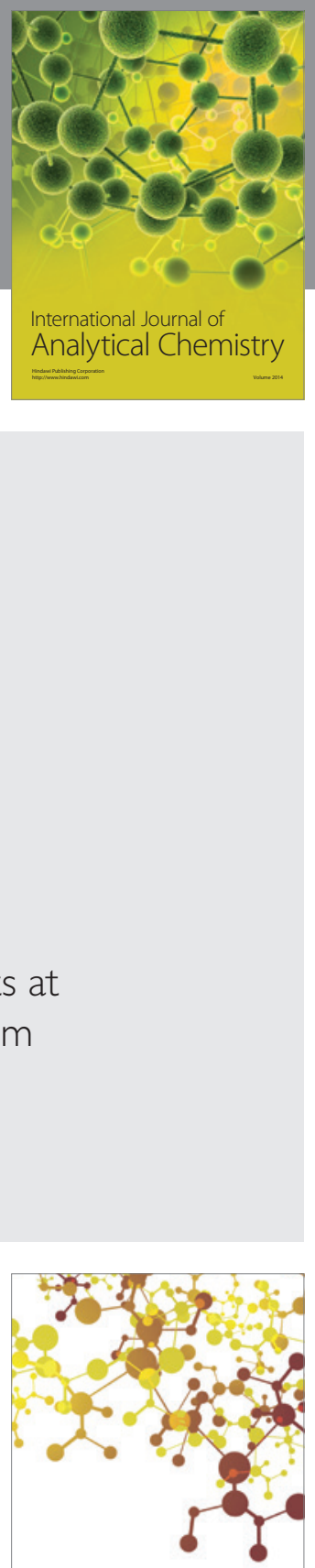

Journal of

Applied Chemistry
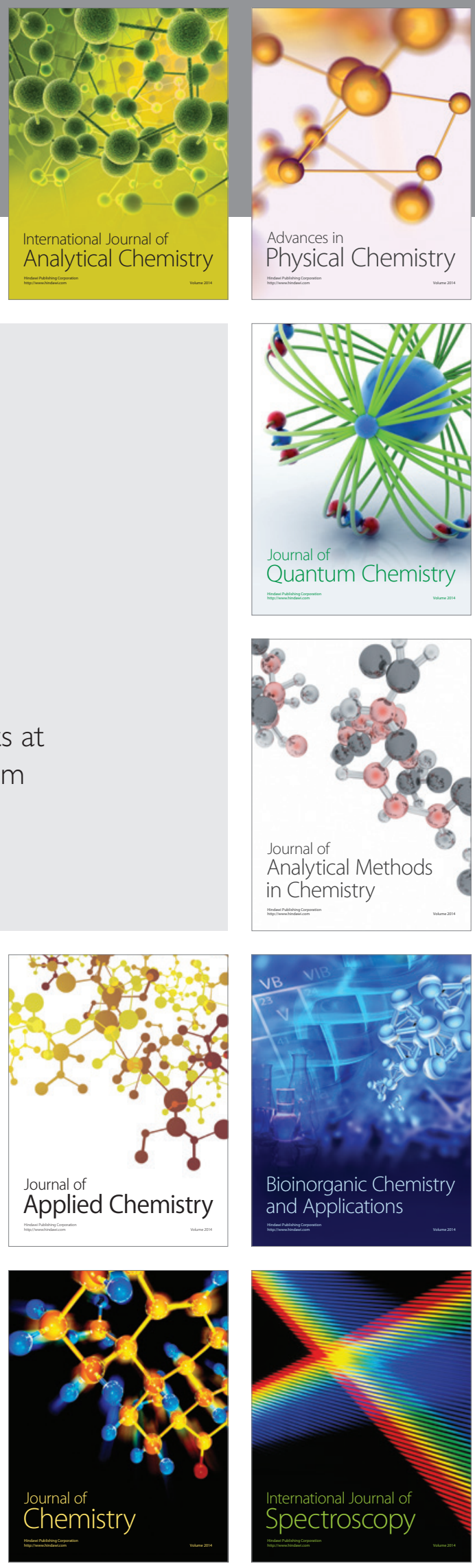